\title{
Study on Extraction and Expression Methods of Bulging Diseases in Ancient City Walls
}

\author{
Song Qing ${ }^{1,2,3, *}$, Hu Yungang ${ }^{1,2,3}$, Hou Miaole ${ }^{1,2,3}$ \\ ${ }^{1}$ School of Geomatics and Urban Spatial Informatics, Beijing University of Civil Engineering and Architecture, 102616 \\ No.15Yongyuan Road, Daxing District, Beijing - (songqing)@stu.bucea.edu.cn; (huyungang, houmiaole) @.bucea.edu.cn \\ ${ }^{2}$ Beijing Key Laboratory For Architectural Heritage Fine Reconstruction \& Health Monitoring, 102616 No.15Yongyuan Road, \\ Daxing District, Beijing \\ ${ }^{3}$ Beijing Advanced Innovation Center for Future Urban Design, Beijing University of Civil Engineering and Architecture, Beijing \\ 100044, China
}

\section{Commission VI, ICWG IV/III, WG VI/4}

KEY WORDS: City wall heritage protection, bulging monitoring, 3D scanning

\begin{abstract}
:
The ancient city wall contains rich cultural values. Due to environmental and human factors, there are many diseases in the ancient city wall: bulging, cracking, etc., which will lead to the collapse or even death of the ancient city wall. Therefore, the monitoring and protection of the ancient city wall is imminent. This paper proposes a new scheme for bulging monitoring for wall bulging. The feature plane is fitted according to the actual scan data, the degree of bulging, the trend and the area size are determined, and the bulging deformation of the city wall is displayed in the form of an image. Simplify workflow, improve data processing efficiency, and display more intuitively
\end{abstract}

\section{INTRODUCTION}

The ancient city wall is one of the important symbols of Chinese civilization, and it is also a powerful testimony of the inheritance of Chinese civilization in history. It has a high historical value and research value. At present, there are still a large number of well-preserved ancient city walls, such as the Forbidden City Wall, Nanjing City Wall and Fuyang City Wall. However, with the passage of time, most of the ancient city walls have been invaded by various degrees from local to whole and some even collapsed, such as the partial collapse of the ancient city wall in Fuyang in 2015. In the past, most of the research on ancient city walls focused on the form, structure and historical and cultural values. There are few studies on the types, causes and grades of ancient city walls (FU,2007; Cui et al., 2015; Zhou., 2018; Meng et al., 2018; Zhou,2018; Zhang et al., 2018; Zhang et al., 2009). In recent years, with the increasing protection of cultural relics in the country, the investigation and protection of cultural relics have received much attention, and relevant research on ancient city walls has also received attention.

In general, there are six types of diseases in the ancient city wall, uneven settlement on the top of the city wall, bulging deformation of the city wall, cracks in the city wall, weathering and spalling of the city wall, destruction of the city wall vegetation and unreasonable use of the city wall (Cao et al., 2013). Among them, the bulging deformation of the city wall is considered to be caused by the poor drainage of the city wall and the infiltration of water into the bauxite inside the city wall (Cao et al., 2010). At the beginning of the bulging, the outer wall skin bulged, and as the degree of bulging intensified, the wall cracked, and eventually the wall peeled off and partially collapsed. Wall bulging is a serious disease with a large potential safety hazard.

At present, there is still no specific research on how to obtain the extent and extent of the bulging of the ancient city wall and how to describe it. The traditional method of measuring points by total station can only obtain discrete point coordinates (Zhou et al., 2011;Zhang Qi, 2011;Li Wei,2018), and the measurement accuracy of the building staff's tape measure is difficult to meet the requirements of range measurement. In addition, after the bulging of the ancient city wall, it is difficult to determine the initial state of the ancient city wall, which brings great difficulty to the determination of the degree of expansion. Therefore, this paper explores how to accurately measure the bulging of the ancient city wall, how to extract the range and size of the bulging, and describe the theory and method. It will provide reliable technical support for disease investigation and disease monitoring of ancient city walls, making the protection of ancient city walls more scientific.

\section{GENERAL IDEAS}

The 3D laser scanner can quickly acquire high-precision 3D point cloud data of scanned objects, and has the characteristics of high precision, high efficiency and non-contact. It is one of the fastest developing technologies in the field of surveying and mapping in recent years (Wang,2010). According to the characteristics of the bulging disease of the ancient city wall, the 3D laser scanner was used for data collection, and the bloating disease information was extracted from the highprecision point cloud data, and the disease information was further expressed. The 3D point cloud data has rich surface information of the object. If the initial state of the ancient city wall can be obtained by the plane fitting algorithm, the current

\footnotetext{
${ }^{*}$ Tel.: +1 8210289095 .

E-mail address: 1269220472@qq.com.
} 
information can be compared with the fitting plane, and the bulging disease of the ancient city wall. The main idea of this paper is shown in Figure 1.

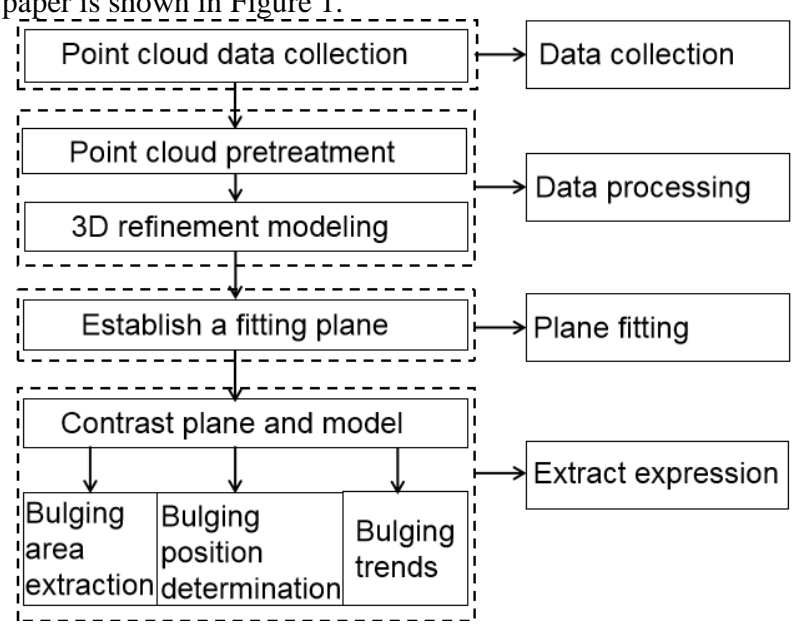

Figure 1. City wall bulging disease deformation information extraction technology route

\section{DATA ACQUISITION AND PROCESSING}

There are many literatures on the use of 3D laser scanners to collect data (Zhou et al., 2008; Cheng, 2012; Wang et al., 2019). Since the city wall is a linear object, when the length of the wall is long, the data accuracy will be affected if the scanning data of each station is spliced without control measurement. To this end according to the distance calibration of the instrument scanner, scanning and splicing at a certain interval to generate a complete point cloud data of a section of the city wall.

Point cloud data requires pre-processing and 3D modeling. First, remove the unnecessary non-target point cloud, then perform denoising to eliminate the isolated noise points, and finally encapsulate the point cloud into an irregular triangulation. The original irregular triangulation has holes, self-intersection and other issues, and it is necessary to repair the triangulation to make it a closed and complete mesh surface, and finally obtain a complete three-dimensional model.

\section{WALL FITTING METHOD}

Plane fitting refers to the process of calculating the required plane from the acquired discrete points (Bo, 2018), usually using eigenvalue method, least squares method, etc. (Acharya et al., 1988; He et al., 2003; Wang et al.,2001; Guan et al., 2008). Due to the surface of the ancient city wall, there are both an inflated portion and a non-bulging portion. If the point cloud data of the entire surface is involved in the fitting algorithm during plane fitting, regardless of the difference between inflation and non-bulging, there will be a large error in the fitted surface. Therefore, when using the above algorithm, the influence of the error cannot be eliminated.

The main principle of the RANSAC algorithm is to input a set of experimental data, repeatedly select a set of random subsets (intra-points) in the data by iteration, and remove those input data (outlier points) that are inconsistent with the estimated parameters, and then pass the correct The input data is used to estimate the parameters ( $\mathrm{Li}$ et al., 2011), giving a model where the maximum probability is applied to the intra-point. The algorithm flow is shown in Figure 2 (Zhou et al., 2011; Cao et al., 2012).

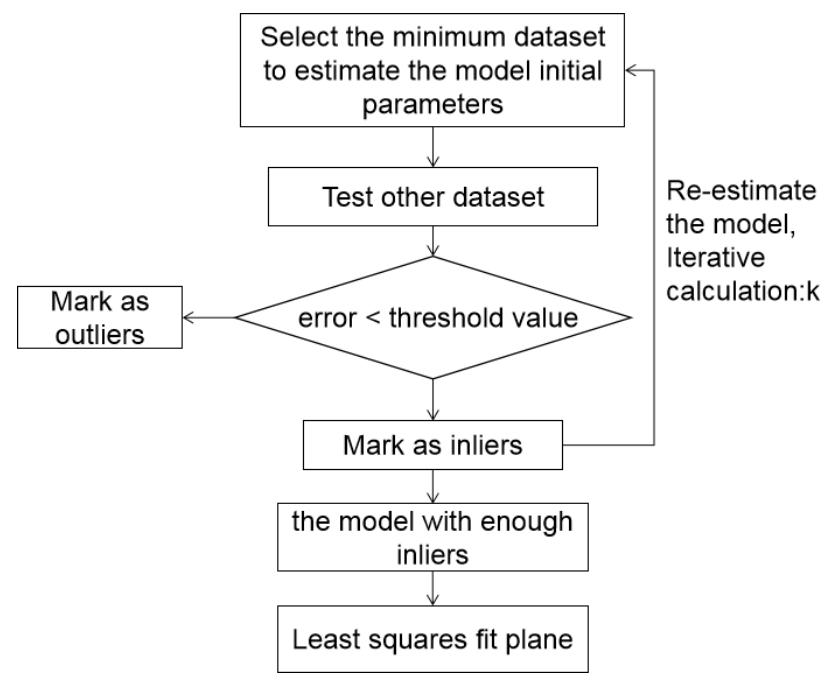

Figure 2 Schematic diagram of the RANSAC algorithm flow

In the calculation process of RANSAC algorithm, the minimum data $n$ required by the model is determined. Because of the need to fit the plane, $n$ takes 3 , and the artificially set parameter

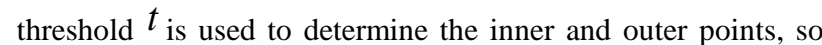
that the model is reasonable enough to minimize the number of inner points $d$ (Yan et al., 2007) The number of repetitions $k$ of performing the above process can be derived from the theoretical results.

Two parameters need to be introduced when determining the $k$ value, $p$ is used to indicate the probability that the points randomly selected from the data set are intra-points during the iterative process, and $w$ is used to indicate the probability of selecting one intra-point from the data set each time:

$$
w=\frac{\text { number of in }- \text { office points }}{\text { number of data sets }}
$$

Suppose the estimation model selects $n$ points as sample points, $w^{n}$ is the probability that all $n$ points are intra-office points; $\left(1-w^{n}\right)$ is the probability that at least one of the $n$ points is an outlier, indicating the data set from the data set. A bad model was estimated. $\left(1-w^{n}\right)^{k}$ indicates that the algorithm will never choose the probability that $n$ points are intra-points, which is the same as $(1-p)$, namely: $1-p=\left(1-w^{n}\right)^{k}$. Take the logarithm on both sides, that is, get the number of iterations:

$$
k=\frac{\lg (1-p)}{\lg \left(1-w^{n}\right)} \Lambda \Lambda \Lambda \Lambda(1)
$$

The selection of the threshold is very important, directly affecting the judgment of the outliers[30]. Because when the effective point is judged, if the selected $t$ is small, the effective point that should be selected is discarded; and if the selected $t$ is large, the abnormal point or the error point may be mistakenly determined as the effective point. Therefore, we 
must weigh the accuracy and efficiency to manually choose the size of the $t$ value (Wei et al., 2014).

\section{DESCRIPTION AND EXPRESSION OF DISEASE INFORMATION OF ANCIENT CITY WALL}

Calculate the point-to-plane distance calculation by spatially analysing the geometry with the fitted reference plane.The general equation of the plane: $A x+B y+C z+D=0$ Where $n=(A, B, C)$ is the normal vector of the plane, and $D$ is the distance required to translate the plane to the origin of the coordinates (so $D=0$, the plane passes the origin).The modulus of the vector (length): Given a vector $V(x, y, z)$, then $|V|=\operatorname{sqrt}\left(x^{2}+y^{2}+z^{2}\right)$.The dot product of the vector (inner product): Given two vectors $V_{1}\left(x_{1}, y_{1}, z_{1}\right)$ and $V_{2}\left(x_{2}, y_{2}, z_{2}\right)$, their inner product is: $V_{1} V_{2}=x_{1} x_{2}+y_{1} y_{2}+z_{1} z_{2}$.Then, the distance $d$ from the point $Q=\left(x_{0}, y_{0}, z_{0}\right)$ to the plane $A x+B y+C z+D=0$ is a point $P(x, y, z)$ connecting $P Q$ in any plane, and a normal vector $\vec{n}=(A, B, C)$ passing through $P$ as a plane.It can be seen that the distance $d$ from the $Q$ to the plane is exactly the projection length of the $P Q$ on the normal vector $\vec{n}$.

Therefrom, we can find the distance of the different points from the fitting plane, and regard the different distances as the magnitude of the bulging, that is, the amount of deviation. Since there is no standard for the classification of bulging degree of the existing city wall disease, it is necessary to set the deviation range as a parameter according to the actual situation and the relevant literature, and analyze it. According to the actual bulging condition, the bulging range is divided by the interval of $1 \mathrm{~mm}$, and an appropriate threshold is established. The different deviations are expressed in different colors, and the thermal change diagram of the wall bulging disease is obtained, which will more intuitively see the process of the wall bulging change. From the thermal map of the wall bulging disease dynamics, we can see that we can get different bulging area distribution according to different deviations. From the different bulging area distribution, we can determine the stage of the wall bulging disease development, so as to judge whether it is the key monitoring period.

According to the thermal map of the dynamic change of the wall bulging disease, the edge extraction algorithm is used to extract the wall changes under different levels, and the dynamic change line drawing of the wall bulging disease can be obtained by accumulating the wall. From the drawing of the dynamic change line of the wall bulging disease, the morphological information such as the origin, expansion direction and development trend of the wall bulging disease can be clearly understood. Through this method, the disease of the wall bulging can be analyzed more comprehensively and accurately. The detailed study of the disease, the design of the wall repair scheme and the repair of the specific wall walls provide clear disease presentation and provide reliable data support for the repair of the city wall.

The Boolean operation is performed by performing a Boolean operation on the 3D model and the fitting plane, and the positioning and area calculation can be performed on the area.

\section{EXPERIMENT AND ANALYSIS}

The scanning area is a long strip-shaped area with a length of about $199 \mathrm{~m}$ on the west side of the ancient city wall. The wall is about $9 \mathrm{~m}$ high. The section is trapezoidal with a narrow upper and a lower width. The corner is about $8.55 \mathrm{~m}$ wide, the top is $6.63 \mathrm{~m}$ wide, and the wall contraction is about $10 \%$ (Cao et al., 2010). The city walls are all made of gray brick, and the construction method is: out-and-in bond. The shedding and collapse of the city wall caused by the disease has been shielded and protected by scaffolding.

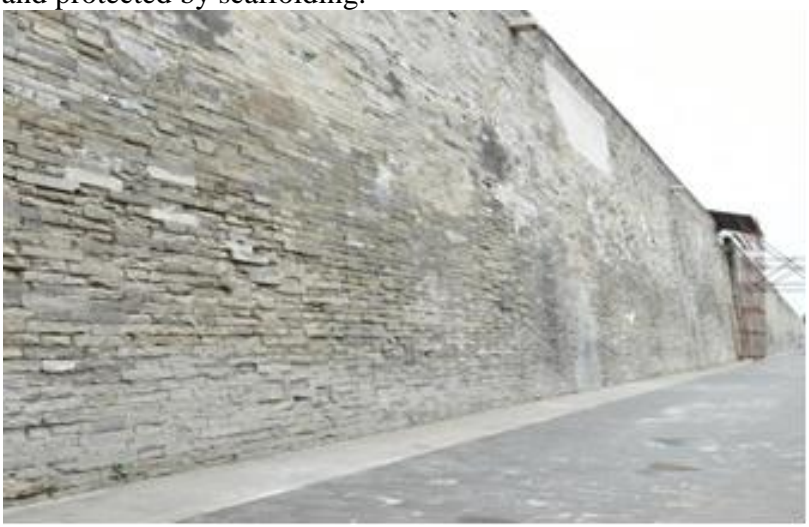

Figure 3 Actual situation of the city wall

According to the external conditions of the 3D scanner, the incident angle should be less than $70^{\circ}$ to set the length of the interval measured by each station, and a total of 13 stations are set (Jia et al., 2018).

Considering the subsequent splicing, there are three common target balls between two adjacent stations. In order to carry out detailed analysis of the city wall, interception is carried out for about $40 \mathrm{~m}$ (the occlusion part is removed), and from left to right, it is the $\mathrm{DE}$ segment of the $\mathrm{CD}$ segment of the $\mathrm{AB}$ segment $\mathrm{BC}$ segment. The results of the field collection are shown in Figure 4 (splicing with a spherical target).

Generating TIN model based on point cloud, Take the BC section of the city wall as an example, As shown in Figure 5.

The fitting plane produces the result, and the point with the plane distance less than 0.06 is considered as the intra-point. Take the BC section of the city wall as an example, The result is shown in Fig. 6. 


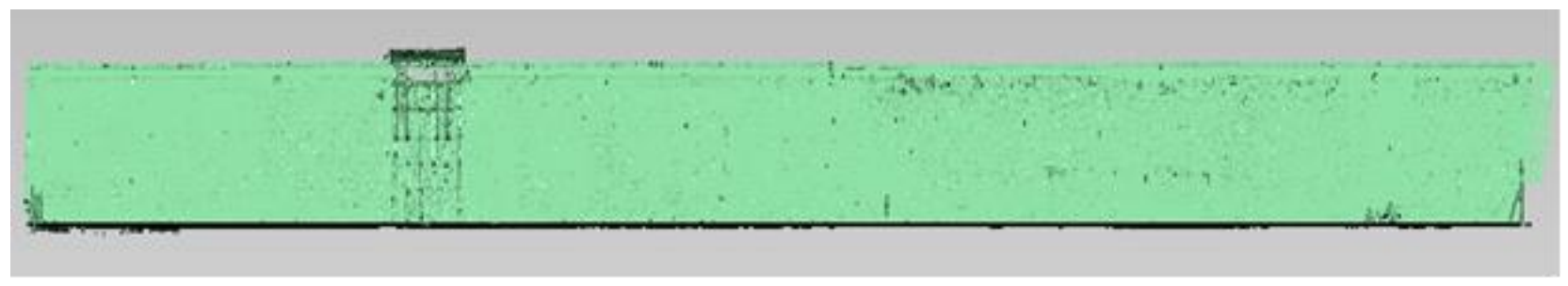

Figure 4 City Wall Scanning Point Cloud (with scaffolding occlusion)

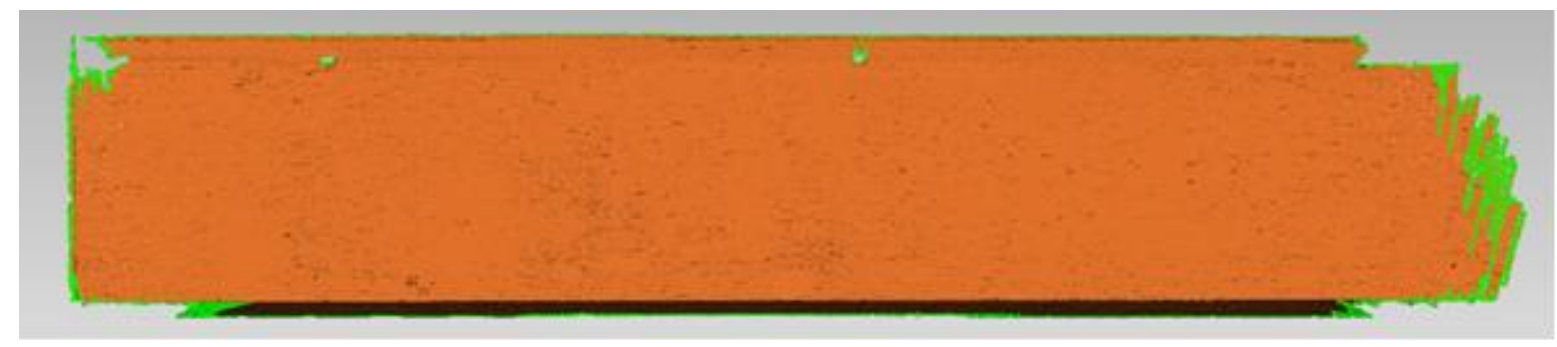

Figure 5 TIN model of the BC section of the city wall

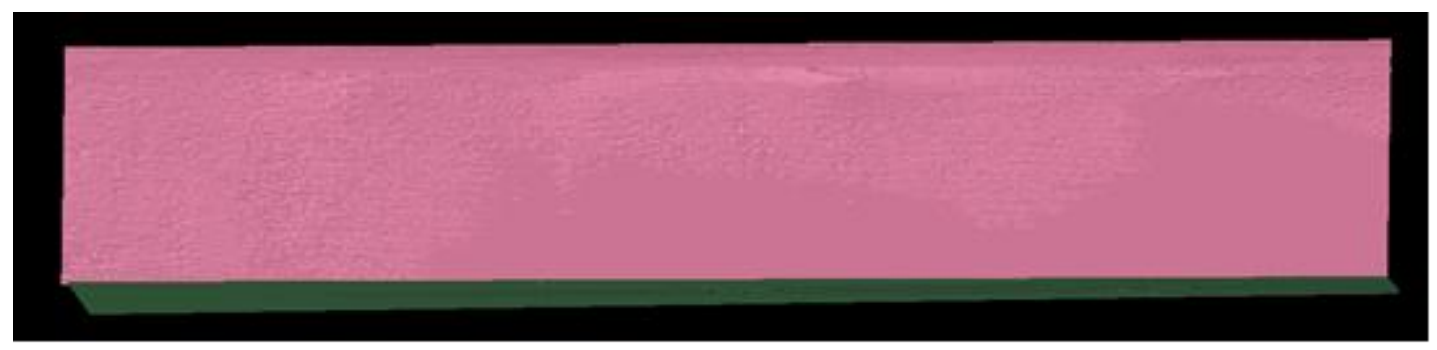

Figure 6 City Wall BC segment fitting plane generation result

Taking the generated fitting plane as the reference plane, set the different distances from the plane to different deviations, and set the deviation values to: $0.1 \mathrm{~m}$ (as shown in Figure a), $0.05 \mathrm{~m}$ (as in Figure b), and $0.02 \mathrm{~m}$ ( As shown in Figure c), $0.01 \mathrm{~m}$ (Figure d), Take the BC section change map of the city wall as an example, as shown in Figure 7.

From Fig. 7, we can see that the wall bulging with the bulging deviation of $0.05 \mathrm{~m} \sim 0.02 \mathrm{~m}$ is the largest, the largest area, and the maximum bulging point is $0.1 \mathrm{~m}$.It is judged by experience that this stage is the mid- or late-stage of bulging development. This level is the stage that needs to be monitored.

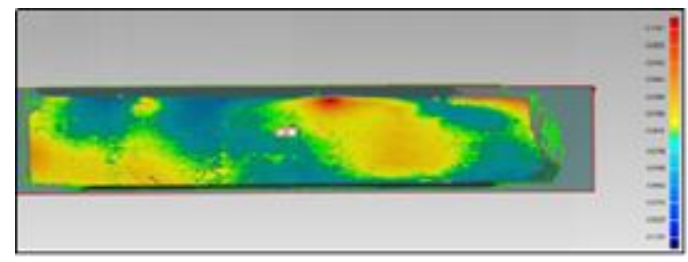

a

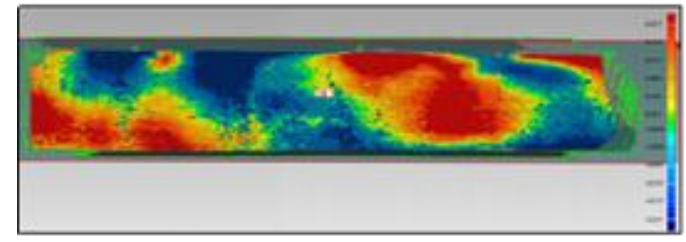

c
According to the dynamic map of the dynamic change of the wall bulging disease, the edge extraction algorithm is used to extract the wall changes under different levels, and after accumulating them, the dynamic change line drawing of the wall bulging disease can be obtained (as shown in Fig. 8). We found that the bulging was mainly concentrated in four areas, and the bulging areas all expanded downward and there was a clear tendency to bulge near some of the drainage ditch.

The wall TIN model and the fitting plane are Boolean operations to obtain an accurate bulging area, which can be used for positioning and area calculation (as shown in Table 1 )
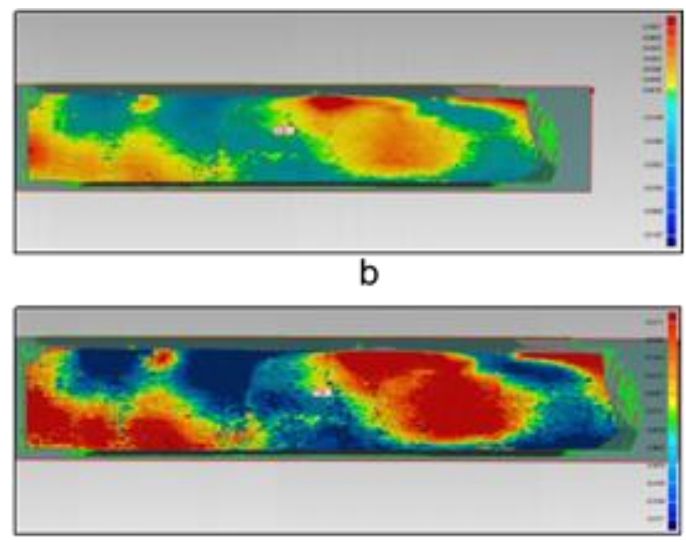

d 
Figure 7 Thermal dynamic diagram of the wall bulging disease

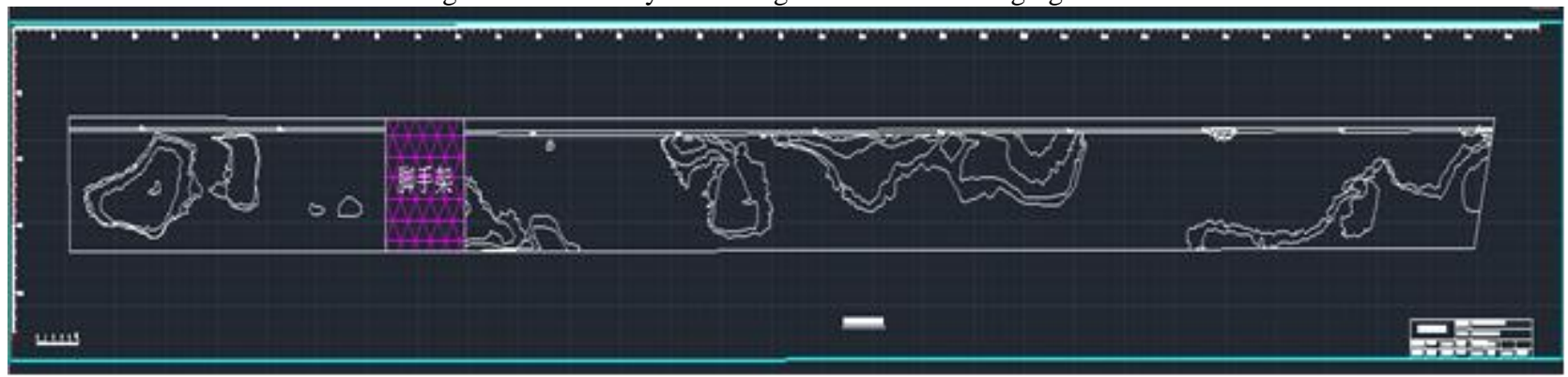

Figure 8 Line drawing of the dynamic change of the wall bulging disease

\begin{tabular}{|c|c|c|c|c|}
\hline Number & Drain port number & Distance & Area & Orientation \\
\hline 1 & 1 & 4.871 & 32.1654 & South-southeast \\
\hline 2 & 4 & 0.4443 & 3.9122 & southeast \\
\hline 3 & 5 & 2.3721 & 3.9625 & Southeast east \\
\hline 4 & 5 & 9.4714 & 2.9496 & Southeast east \\
\hline 5 & 6 & 1.5131 & 0.3595 & West \\
\hline 6 & 7 & 3.4155 & 12.4856 & West-southwest \\
\hline 7 & 7 & 3.2194 & 45.2205 & south \\
\hline 8 & 9 & 2.3329 & 6.3567 & east \\
\hline 9 & 10 & 5.3606 & 4.6014 & South-southeast \\
\hline 10 & 11 & 2.8234 & 1.6308 & $\begin{array}{c}\text { Symmetrical distribution } \\
\text { on both sides }\end{array}$ \\
\hline 11 & & & 1.7429 & South-southeast \\
\hline
\end{tabular}

Table 1 Statistics on the wall bulging disease information

\section{CONCLUSION}

Wall monitoring is a dynamic process, so in the process of wall protection should consider its development in the long term, dig deep into the history of the wall, pay attention to the daily monitoring of the wall. As an important work in the daily monitoring and maintenance of wall, the monitoring of wall bulging needs to be more effectively monitored and intuitively expressed by the technical means. This paper discusses the new application of 3D laser scanning technology in the monitoring data processing and expression of wall bulging deformation. Compared with the existing methods for monitoring the bulging deformation, which consumea large amount of human and financial resources, single point data, 3D laser scanning for the preservation of ancient relics, not only reduce the workload of the field, but also has the advantages of high precision, high efficiency, can completely replace the current part of the mapping method. Moreover, 3D laser scanning can obtain a large number of data and intuitive appearance morphological changes can also be extracted by Boolean calculation of the change characteristics of analysis, in the form of deviation chromatography to achieve a vivid and visual display of wall deformation monitoring data. It is also a trend of wall monitoring in the future to study the visual and effective expression of wall bulging monitoring data into chartform.

\section{REFERENCES}

FU Xiaoyu. (2007). Exploring Chinese ancient city wall protection. (Doctoral dissertation, Beijing Forestry University).

Cui Biao Fu Zhengrong, Liu Xiaobin, Liang Lijun, \& Zhang Bingjian. (2015). Taozhu city walls disease Preliminary research Discussion and protection measures. Cultural Relics Protection and Archaeology, 27(3), 8-13.

Zhou Yuanqiang. (2018). A preliminary study on the influencing factors of an ancient city wall disease in the west. (Doctoral dissertation, Xi'an University of Technology).

Meng Qingxu, Li Hanxiao. (2018). Study on the anatomical structure of the Liao Dynasty city wall. China City Wall (00), 66-73. 
Zhou Yuan. (2018). A review of the study of Nanjing Mingcheng Wall in the past forty years. China City Wall (00), 33-45.

Zhang Dandan, \& Wang Bing. (2018). On the protection and restoration of the Juyongguan city wall in Beijing. Research on heritage and protection (11).

Zhang Wenge, Xi Xiangdong. (2009). Stability Analysis of Pingyao Ancient City Wall. Building Structure (3), 110-112.

Cao Xiaoli, Li Deshan, \& Liu Weihua. (2013). Investigation and protection of the current status of the Forbidden City Wall. The Palace Museum (1).

Cao Xiaoli, \& Li Deshan. (0). Investigation on the current situation of the Forbidden City Wall. The 85th Anniversary of the Palace Museum and the 7th Academic Symposium of the China Forbidden City Society.

Zhou Jie, Hu Wusheng, \& Zhu Mingchen. (2011). Nanjing City Wall Bulging Monitoring and Data Processing Method. Modern Surveying and Mapping, 34(5), 11-12.

Zhang Qi. (2011). Analysis of the monitoring of the wall of the Lion Rock section in Nanjing. Jiangsu Architecture (2), 28-30.

Li Wei. (2018). Fengyang Gulou City Wall Bulging Monitoring and Analysis. Shanxi Architecture.

Wang Xiaoxiao. (2010). Ground 3D laser scanning modeling and its application in building surveying and mapping. (Doctoral dissertation, Central South University).

Zhou Junzhao, Zheng Shumin, Hu Song, \& Zhou Jianbo. (2008). Application of Ground 3D Laser Scanning in the Protection and Mapping of Grottoes in Stone Grottoes. Bulletin of Surveying and Mapping, 11(12), 68-69.

Cheng Huwei. (2012). Precision mapping of ancient city wall based on 3D laser scanning technology [N]. China Cultural Relics, (007)

Wang Min, Du Weiji, \& Duan Wei. (2019). Reconstruction and application analysis of civil air defense model based on 3D laser scanning technology. Bulletin of Surveying and Mapping (5), 148-151.

Bo Huaizhi. (2018). Accuracy comparison and analysis of three point cloud data plane fitting methods. Mapping and spatial geographic information, v.41; No.229(05), 216-218.

Acharya, P. K. , \& Henderson, T. C. . (1988). Parameter estimation and error analysis of range data. IEEE International Conference on Robotics \& Automation. IEEE Xplore.

He Wenfeng, \& Cha Hongbin. (2003). Depth image registration based on planar features. National Academic Annual Meeting of China Artificial Intelligence Society.

Wang, C., Tanahashi, H., Hirayu, H., Niwa, Y., \& Yamamoto, K. (2001). Comparison of Local Plane Fitting Methods for Range Data. IEEE Computer Society Conference on Computer Vision \& Pattern Recognition.
Guan Yunlan, Cheng Xiaojun, \& Shi Guigang. (2008). A Robust Point Cloud Data Plane Fitting Method. Journal of Tongji University (Natural Science Edition), 36(7), 981-984.

Li Na, Ma Yiwei, Yang Yang, Gao Lili, (2011). Using the ransac algorithm to perform point cloud segmentation on building facades. Surveying and Mapping Science (5).

Zhou Chunlin, Zhu Hehua, \& Li Xiaojun. (2011). Random sampling consistency plane fitting and its application research. Computer Engineering and Applications, 47(7), 177-179.

Cao Wei, Feng Ying, Yang Yuntao, \& Chen Wei. (2012). Application of Ransac Planar Estimation Algorithm in Pavement Object Volume Measurement. Journal of Transducer Technology, 25(3), 413-416.

Yan Kun, Wang Yixiu, \& Ge Mei. (2007). Research on measurement robot automatic monitoring system for deformation monitoring. Geospatial information, 5(6), 121-123.

Wei Yingzi, \& Liu Xiaoli. (2014). Robust point cloud plane fitting based on random extraction consistency. Journal of Beijing University of Technology, 40(3), 400-403.

Feng C, Taguchi Y, Kamat V Method for extracting planes from 3D point cloud sensor data[J]. 2016.

Hua Qiancheng, Xu Maolin, Yang Fengqi, \& Dong Cuijun. (2014). Research on measuring robot slope monitoring system based on tm30. Beijing Surveying and Mapping (4), 60-62.

Jia Dongfeng, Cheng Xiaojun, Liu Yanping, \& Wang Feng. (2018). Analysis of the influence of incident angle on the precision of laser point cloud. Engineering Investigation, 46(5), 43-49. 